Pacific Journal of Mathematics

ATTACHING HUREWICZ FIBRATIONS WITH FIBER 


\section{ATTACHING HUREWICZ FIBRATIONS WITH FIBER PRESERVING MAPS}

JAMES E. ARNOLD, JR.

When working with fibrations, there are times when standard topological constructions involving identifications are useful. The problem of course, is to show that identifying fibrations in the proper way yields a fibration. This paper establishes a fairly general result concerning attaching Hurewicz fibrations over a fixed base space with a fiber preserving map. This can be applied to obtain many common topological constructions. In particular a theorem of P. Tulley on mapping cylinders is strengthened, which in turn strengthens the main theorems on strong fiber homotopy equivalence and extensions of fibrations obtained by $P$. Tulley and $S$. Langston. In addition, these results are applied to obtain a stronger version of Dold's pasting lemma, an important step in the construction of classifying spaces for fibrations.

\section{Definitions and notation.}

By a space over $B$ we will mean a triple $\xi=(E, p, B)$ where $p: E \rightarrow B$ is a map. We will often refer to $E$ as the total space, and $B$ as the base space of $\xi$. If $A \subset B$ we will let $\xi_{A}=\left(E_{A}, p_{A}, A\right)=$ $\left(p^{-1}(A), p \mid p^{-1}(A), A\right)$. Given two spaces over $B, \xi=(E, p, B)$ and $\xi^{\prime}=\left(E^{\prime}, p^{\prime}, B\right)$ we will denote by $f: \xi \rightarrow \xi^{\prime}$ a fiber preserving map from $\xi$ to $\xi^{\prime}$, i.e. a map $f: E \rightarrow E^{\prime}$ such that $p^{\prime} f=p$. Two such maps $f, g: \xi \rightarrow \xi^{\prime}$ are said to be fiber homotopic denoted $f \sim g: \xi \rightarrow \xi^{\prime}$ if there is a homotopy $H$ from $f$ to $g$ such that for each $t \in I, p^{\prime} H(e, t)=$ $p(e) . \quad \xi$ is fiber homotopy equivalent to $\xi^{\prime}$, denoted $\xi \sim \xi^{\prime}$, if there are fiber preserving maps $f: \xi \rightarrow \xi^{\prime}$ and $g: \xi^{\prime} \rightarrow \xi$ with $f g$ and $g f$ fiber homotopic to the respective identity maps.

A space over $B$ will be called a Hurewicz fibration, or more simply a fibration if it has the universal covering homotopy property, or equivalently has a lifting function $\lambda$. Recall that a lifting function for $\xi=(E, p, B)$ is a section for $\left(E^{I}, \widetilde{p}, \Omega_{p}\right)$, where

$$
\Omega_{p}=\left\{(e, \omega) \in E \times B^{I} \mid p(e)=\omega(0)\right\}
$$

and $\tilde{p}(\omega)=(\omega(0), p \omega)$. (i.e. a map $\lambda: \Omega_{p} \rightarrow E^{I}$ with $\lambda(e, \omega)(0)=e$ and $p \lambda(e, \omega)(t)=\omega(t)$.

A lifting function is regular if it takes constant paths in the base space to constant paths in the total space, and if a fibration $\xi$ has a regular lifting function, $\xi$ will be called a regular fibration. 
Definition (1.1). A slicing function for $\xi_{A}$ is a map $\varphi: E_{A} \times A \rightarrow E_{A}$ such that $\varphi(e, p(e))=e$ and $p \varphi(e, \alpha)=a$.

Note that the existence of a slicing function for $\xi_{A}$ implies $\xi_{A}$ is a regular fibration. (A regular lifting function is defined by $\lambda(e, \omega)(t)=$ $\varphi(e, \omega(t))$.) For our purposes slicing functions are generally easier to work with than lifting functions since the product topology is simpler than the compact open topology of function spaces.

Definition (1.2). A pair of spaces over $B\left(\xi, \xi_{0}\right)$, is a pair of triples $\xi=(E, p, B), \xi_{0}=\left(E_{0}, p_{0}, B\right)$ such that $E_{0} \subset E$ and $p_{0}=p \mid E_{0}$.

If $\xi$ and $\xi_{0}$ are both fibrations $\left(\xi, \xi_{0}\right)$ will be called a pair of fibrations. If in addition there is a lifting function $\lambda: \Omega_{p} \rightarrow E^{I}$ for $\xi$ such that $\lambda \mid \Omega_{p_{0}}$ is a lifting function for $\xi_{0}$, then $\left(\xi, \xi_{0}\right)$ will be called a fibered pair.

Finally, we will make frequent use of cofibrations.

Definition (1.3). $(X, A)$ is a cofibration if any partial homotopy $\bar{H}: X \times\{0\} \cup A \times I \rightarrow Y$ has an extension $H: X \times I \rightarrow Y$.

If in addition $A$ is closed we will say $(X, A)$ is a closed cofibration. If $X$ is a Hausdorff space all cofibrations $(X, A)$ are closed.

2. Preliminary lemmas and the main theorem.

Lemma (2.1). ( $X, A)$ is a closed cofibration if and only if there are maps $D: X \times I \rightarrow X, \phi: X \rightarrow I$ with the following properties:

(a) $D(x, 0)=x$ for all $x \in X$

(b) $D(a, t)=a$ for all $a \in A, t \in I$

(c) $A=\phi^{-1}(1)$ and $D\left(\phi^{-1}(0,1] \times 1\right) \subset A$.

The proof, which we will omit, is an exercise in [7], p. 57. The proof is essentially the same as that in [11].

Lemima (2.2). If $\left(\xi, \xi_{0}\right)$ is a regular fibered pair, then $\left(E, E_{0}\right)$ is a closed cofibration if and only if there are maps $D: E \times I \rightarrow E$ and $\alpha: E \rightarrow I$ with the following properties:
(a) $D(e, 0)=e$ for all $e \in E$
(b) $D(e, t)=e$ for all $e \in E_{0}, t \in I$
(c) $\alpha^{-1}(1)=E_{0}$
(d) $\quad D\left(\alpha^{-1}(0,1] \times 1\right) \subset E_{0}$
(e) $p D(e, t)=p(e)$ for all $e \in E, t \in I$.

Note that the only difference between Lemmas (2.1) and (2.2) is part (e), which says that $D$ moves points along fibers.

Proof. Define $D(e, t)=\lambda\left[D^{\prime}(e, t), \omega(e, t)\right](1)$ and $\alpha(e)=\dot{\phi}(e)$, where $D^{\prime}$ and $\phi$ are as in Lemma $(2.1), \omega(e, t)$ is the path given by $\omega(e, t)(s)=$ 
$p D^{\prime}(e,(1-s) \cdot t)$, and $\lambda$ is a regular lifting function for $\xi$ whose restriction to $\Omega_{p_{0}}$ is a lifting function for $\xi_{0}$. Property (b) is a consequence of the regularity of $\lambda$, and (d) is a consequence of $\lambda \mid \Omega_{p_{0}}$ being a lifting function for $\xi_{0}$.

Let $\Delta B=\{(b, b) \in B \times B\}$ denote the diagonal of $B$ in $B \times B$. The main theorem will be proven for fibrations with paracompact, locally compact base spaces $B$ such that $(B \times B, \Delta B)$ is a closed cofibration. (ANR's for example satisfy this last condition.) If $B$ is metric then this condition is equivalent to $B$ ULC (uniformly locally contractible). The following lemma in fact concludes that all such spaces are ULC.

Lemma (2.3). If $(B \times B, \Delta B)$ is a closed cofibration then there is a neighborhood $U$ of $\Delta B$ and a map $\sigma: U \rightarrow X^{I}$ such that

(a) $\sigma(x, y)(0)=x$

(b) $\sigma(x, y)(1)=y$

(c) $\sigma(x, x)(t)=x$ for all $t \in I$.

Proof. Choose $D$ and $\phi$ as in Lemma (2.1) and define

$$
\sigma(x, y)(t)=\left\{\begin{array}{l}
\pi_{1} D(x, y, 2 t) \quad 0 \leqq t \leqq \frac{1}{2} \\
\pi_{2} D(x, y, 2-2 t) \quad \frac{1}{2} \leqq t \leqq 1
\end{array}\right.
$$

where $\pi_{i}: B \times B \rightarrow B$ is projection on the $i$ th coordinate $i=1,2$ and $(x, y) \in U=\phi^{-1}(0,1]$.

The next lemma will allow us a more satisfactory statement of the main theorem.

LEMMA (2.4). Let $\left(\xi, \xi_{0}\right)$ be a pair of spaces over $B$ with $\left(E, E_{0}\right)$ a closed cofibration. Then $\left(\xi, \xi_{0}\right)$ is a fibered pair if and only if $\left(\xi, \xi_{0}\right)$ is a pair of fibrations.

The proof of this lemma, which we will omit, consists of choosing a lifting function $\lambda_{0}$ for $\xi_{0}$ and extending it to a lifting function for $\xi$. This can be done exactly as in the Lifting Extension Theorem in [1], replacing $\lambda_{A}$ and $\Omega_{p_{A}}$ by $\lambda_{0}$ and $\Omega_{p_{0}}$ throughout the proof, and noting that $\left(E, E_{0}\right)$ a closed cofibration implies $\left(\Omega_{p}, \Omega_{p_{0}}\right)$ is a closed cofibration. In fact if we let $\pi_{1}: \Omega_{p} \rightarrow E$ be projection on the first coordinate, then $\Gamma=\left(\Omega_{p}, \pi_{1}, E\right)$ is a fibration ( $\Gamma=$ the pullback by $p$ of the standard path fibration over $B)$, and since $\Omega_{p_{0}}=\pi_{1}^{-1}\left(E_{0}\right)$, $\left(\Omega_{p}, \Omega_{p_{0}}\right)$ is a closed cofibration by Theorem 12 of [8].

We come now to the main theorem. If $\left(\xi, \xi_{0}\right)$ is a pair of spaces 
over $B, \eta$ a space over $B$ and $f: \xi_{0} \rightarrow \eta$ a fiber preserving map, we let $\xi \cup_{f} \eta=\left(E \cup_{f} E^{\prime}, q, B\right)$ where $\xi=(E, p, B), \eta=\left(E^{\prime}, p^{\prime}, B\right)$ and $q$ is the map induced by $p$ and $p^{\prime}$.

THEOREM (2.5). Let $B$ be a paracompact, locally compact space such that $(B \times B, \Delta B)$ is a closed cofibration, and $\left(\xi, \xi_{0}\right)$ a pair of fibrations over $B$ with $\left(E, E_{0}\right)$ a closed cofibration. Then for any fibration $\eta$ over $B$ and $f: \xi_{0} \rightarrow \eta,\left(\xi \cup_{f} \eta, \eta\right)$ is a fibered pair.

Proof. Since $(B \times B, \Delta B)$ is a closed cofibration, we can define $d: B \times B \rightarrow I$ by $d=1-\phi$, ( $\phi$ as in Lemma 2.1) with $d^{-1}(0)=\Delta B$. By Lemma (2.4), there is a lifting function $\lambda$ for $\xi$ whose restriction to $\Omega_{p_{0}}$ is a lifting function for $\xi_{0}$. We may assume, in addition, that $\lambda$ is regular, and that there is a regular lifting function $\lambda^{\prime}$ for $\eta$. The proof is the same as in [4], using $d: B \times B \rightarrow I$ instead of a metric.

Since $B$ is paracompact, the Uniformation Theorem applies (see [4]) and thus we need only show any point has a neighborhood $W$ such that $\left(\left(\xi \cup_{f} \eta\right)_{W}, \eta_{W}\right)$ is a fibered pair. We choose $\eta$ and $\sigma$ to be as in Lemma (2.3), and given a point $b_{0} \in B$ choose a neighborhood $V$ of $b_{0}$ with $V \times V \subset U$. We then have slicing functions $\phi$ and $\psi$ for $\xi_{V}$ and $\eta_{V}$ respectively defined as follows:

$$
\begin{array}{cl}
\phi(e, x)=\lambda[e, \sigma(p(e), x)](1) & (e, x) \in E_{V} \times V \\
\psi\left(e^{\prime}, x\right)=\lambda^{\prime}\left[e^{\prime}, \sigma\left(p^{\prime}\left(e^{\prime}\right), x\right)\right](1) & \left(e^{\prime}, x\right) \in E_{V}^{\prime} \times V .
\end{array}
$$

It is important to note here that if $e \in E_{0}, \phi(e, x) \in E_{0}$ for all $x \in V$.

We now let $\theta$ be a neighborhood of $\left(b_{0}, b_{0}\right)$ in $U$ which has the property that $\sigma(x, y)(t) \in V$ for all $(x, y) \in \theta$ and all $t \in I$. There is such a neighborhood since $\sigma$ is continuous and $\sigma\left(b_{0}, b_{0}\right)$ is the constant path at $b_{0}$. Finally we choose a neighborhood $W$ of $b_{0}$ with compact closure such that $\bar{W} \times \bar{W} \subset \theta \subset U . \quad \bar{W}$ then the property that for $e \in E_{\bar{W}}$, $e^{\prime} \in E^{\prime}{ }_{W}, x \in \bar{W}$ and $t \in I$, the functions

$$
\Phi(e, \sigma(p(e), x)(t)) \quad \text { and } \quad \Psi\left(e^{\prime}, \sigma\left(p\left(e^{\prime}\right), x\right)(t)\right)
$$

are defined.

Let $D$ and $\alpha$ be defined for the closed cofibration $\left(E, E_{0}\right)$ as in Lemma (2.2) using the lifting function $\lambda$, and $\mu: E_{\bar{W}} \cup E_{\bar{W}}^{\prime} \rightarrow\left(E \cup_{f} E^{\prime}\right)_{\vec{W}}$ be the identification map. Given $D, \alpha, \mu, \phi, \psi, d$ and $\bar{W}$ as above, we now define a map $\bar{\chi}:\left(E_{\bar{W}} \cup E_{\bar{W}}^{\prime}\right) \times \bar{W} \rightarrow\left(E \cup_{f} E^{\prime}\right)_{\bar{W}}$ which will induce the slicing function $\chi$ for $\left(\xi \cup_{f} \eta\right)_{W}$. To simplify the notation in the definition of $\bar{\chi}$ we will let $\hat{d}=d(p(e), z)$, and $\hat{\sigma}(t)=\sigma(p(e), z)(t)$. 
$\bar{\chi}\left(e^{\prime}, z\right)=\mu\left(\psi\left(e^{\prime}, z\right)\right)$ if $e^{\prime} \in E_{\bar{W}}^{\prime}$ and for $e \in E_{\bar{W}}^{\prime}$ is given by the following formula:

$$
\begin{aligned}
& \bar{\chi}(e, z)=\left\{\begin{array}{cc}
\mu\{\phi[D(e, \hat{d}), z]\} \text { if } 0 \leqq \alpha(e) \leqq 1 / 8 \\
\mu\{\phi[D(e, 8 \alpha(e) \cdot \hat{d}), z]\} \text { if } 1 / 8 \leqq \alpha(e) \leqq 1 / 4 \text { and } \\
0 \leqq 4 \alpha(e) \cdot \hat{d} \leqq 1 / 2 \\
\mu\{f[\phi(D(e, 1), z)]\} \text { if } 1 / 8 \leqq \alpha(e) \leqq 1 / 4 \text { and } \\
1 / 2 \leqq 4 \alpha(e) \cdot \hat{d} \leqq 1 \\
\mu\{\phi[D(e, 2 \hat{d}), z]\} \text { if } 1 / 4 \leqq \alpha(e) \leqq 1 / 2 \text { and } 0 \leqq \hat{d} \leqq 1 / 2 \\
\mu\{\psi[f(\phi[D(e, 1), \hat{\sigma}(2-4 \alpha(e)+(4 \alpha(e)-1) / 2 \hat{d})]), z]\} \text { if } \\
1 / 4 \leqq \alpha(e) \leqq 1 / 2 \text { and } 1 / 2 \leqq \hat{d} \leqq 1
\end{array}\right. \\
& \mu\{\phi[D(e, \hat{d} /(1-\alpha(e))), z]\} \quad \text { if } 1 / 2 \leqq \alpha(e) \leqq 1, \alpha(e) \neq 1 \text { and } \\
& 0 \leqq \hat{d} \leqq 1-\alpha(e) \\
& \mu\{\psi[f(\phi[D(e, 1), \hat{\sigma}((1-\alpha(e)) / \hat{d})]), z]\} \quad \text { if } 1 / 2 \leqq \alpha(e) \leqq 1 \text {, } \\
& \widehat{d} \neq 0 \text { and } 1-\alpha(e) \leqq \hat{d} \leqq 1 \\
& \mu(e) \text { if } \alpha(e)=1 \text { and } \hat{d}=0 \text {. }
\end{aligned}
$$

The function $\bar{\chi}:\left(E_{\bar{W}} \cup E_{\bar{W}}^{\prime}\right) \times \bar{W} \rightarrow\left(E \cup_{f} E^{\prime}\right)_{\bar{W}}$ is continuous, and has the property that for $e \in E_{0}$,

$$
\begin{aligned}
\bar{\chi}(e, z) & =\mu\{\psi[f(\phi(e, p(e)), z]\} \\
& =\mu\{\psi[f(e), z]\} \\
& =\bar{\chi}(f(e), z)
\end{aligned}
$$

Since $\bar{W}$ is compact, $\left(E \cup_{f} E^{\prime}\right)_{\bar{W}} \times \bar{W}$ has the identification topology induced by the map $\mu \times 1:\left(E_{\bar{W}} \cup E_{\bar{W}}^{\prime}\right) \times \bar{W} \rightarrow\left(E \cup \cup_{f} E^{\prime}\right)_{\bar{W}} \times \bar{W}$ (see Lemma 4 of [10]). Thus since $\bar{\chi}(e, z)=\bar{\chi}(f(e), z)$ for $e \in E_{0}, \chi$ induces a map $\chi:\left(E \cup_{f} E^{\prime}\right)_{\bar{W}} \times \bar{W} \rightarrow\left(E \cup_{f} E^{\prime}\right)_{\bar{W}}$. To complete the proof we need only check that $\chi$ is a slicing function for $\left(\xi \cup_{f} \eta\right)_{\bar{w}}$. We leave these details to the reader. Also we have claimed that $\left(\xi \cup_{f} \eta, \eta\right)$ is a fibered pair. To see this note that the slicing function $\chi$ when restricted to $E_{\bar{W}}^{\prime} \times \bar{W}$ is a slicing function for $\eta_{\bar{W}}$. Thus the local lifting functions defined by these slicing functions will be lifting functions of pairs, and the Uniformization Theorem will give us a lifting function for $\xi U_{f} \eta$ whose restriction is a lifting function for $\eta$.

Before considering applications of Theorem (2.5), the natural question arises as to whether or not the fiber homotopy type of the fibrations we obtain depends only on the fiber homotopy class of the attaching map. An affirmative answer is provided by the next theorem.

Definition (2.6). We will say a pair of spaces $\left(\xi, \xi_{0}\right)$ over $B$ is a closed cofibration if we can define maps $D: E \times I \rightarrow E$ and $\alpha: E \rightarrow I$ 
satisfying the properties given in Lemma (2.2).

Lemma (2.2) then shows that a pair of fibrations $\left(\xi, \xi_{0}\right)$ is a closed cofibration if and only if $\left(E, E_{0}\right)$ is a closed cofibration.

THEOREM (2.7). Let $\left(\xi, \xi_{0}\right)$ be a closed cofibration. Then if $f \sim g: \xi_{0} \rightarrow \eta, \xi \cup_{f} \eta$ is fiber homotopy equivalent to $\xi \cup_{g} \eta$.

Note that we do not assume $\xi$ or $\xi_{0}$ are fibrations. Also note that all maps and homotopies involved in the proof of the theorem leave $\eta$ fixed. One could state this as a relative theorem, making the proper definitions of relative fiber homotopies etc.

Proof. Let $G: E_{0} \times I \rightarrow E^{\prime}$ be a fiber homotopy with $G(e, 0)=f(e)$, $G(e, 1)=g(e)$, and let $D: E \times I \rightarrow E, \alpha: E \rightarrow I$ be as in Definition (2.7). Let $\mu: E \cup E^{\prime} \rightarrow E \cup_{f} E^{\prime}$ and $\nu: E \cup E^{\prime} \rightarrow E \cup_{g} E^{\prime}$ denote the identification maps. We define $\bar{h}: E \cup E^{\prime} \rightarrow E \cup_{g} E^{\prime}$, and $\bar{h}^{\prime}: E \cup E^{\prime} \rightarrow E \cup_{f} E^{\prime}$ as follows:

$$
\begin{aligned}
& \bar{h}(e)=\left\{\begin{array}{lll}
\nu[D(e, 2 \alpha(e))] & \text { if } e \in E \text { and } 0 \leqq \alpha(e) \leqq 1 / 2 \\
\nu[G(D(e, 1), 2-2 \alpha(e))] & \text { if } e \in E \text { and } 1 / 2 \leqq \alpha(e) \leqq 1 \\
\nu(e) & \text { if } e \in E^{\prime},
\end{array}\right. \\
& \bar{h}^{\prime}(e)=\left\{\begin{array}{lll}
\mu[D(e, 2 \alpha(e))] & \text { if } e \in E \text { and } 0 \leqq \alpha(e) \leqq 1 / 2 \\
\mu[G(D(e, 1), 2 \alpha(e)-1] & \text { if } e \in E \text { and } 1 / 2 \leqq \alpha(e) \leqq 1 \\
\mu(e) & \text { if } e \in E^{\prime} .
\end{array}\right.
\end{aligned}
$$

$\bar{h}$ and $\bar{h}^{\prime}$ are continuous and induce fiber preserving maps $h: E \cup_{f} E^{\prime} \rightarrow$ $E \cup_{g} E^{\prime}$ and $h^{\prime}: E \cup_{g} E^{\prime} \rightarrow E \cup_{f} E^{\prime}$ respectively.

We define $\bar{H}:\left(E \cup E^{\prime}\right) \times I \rightarrow E \cup_{f} E^{\prime}$ and $\bar{K}:\left(E \cup E^{\prime}\right) \times I \rightarrow E \cup_{g} E^{\prime}$ which induce fiber preserving homotopies $H:\left(E \cup_{f} E^{\prime}\right) \times I \rightarrow E \cup_{f} E^{\prime}$ from the identity map to $h^{\prime} h$ and $K:\left(E \cup_{g} E^{\prime}\right) \times I \rightarrow E \cup_{g} E^{\prime}$ from the identity map to $h h^{\prime}$ by the following formulas:

$$
\bar{H}(e, t)=\left\{\begin{array}{c}
\mu[D(D(e, 2 \alpha(e) \cdot t), 2 \alpha(D(e, 2 \alpha(e) \cdot t)) \cdot t)] \\
\text { if } e \in E, 0 \leqq \alpha(e) \cdot t \leqq 1 / 2 \text { and } \\
0 \leqq \alpha(D(e, 2 \alpha(e) \cdot t)) \cdot t \leqq 1 / 2 \\
\mu[G\{D[D(e, 2 \alpha(e) \cdot t), 1], 2 \alpha(D(e, 2 \alpha(e) \cdot t)) \cdot t-1] \\
\text { if } e \in E, 0 \leqq \alpha(e) \cdot t \leqq 1 / 2 \text { and } \\
1 / 2 \leqq \alpha(D(e, 2 \alpha(e) \cdot t \leqq 1 \\
\mu[G(D(e, 1),(2-2 \alpha(e)) \cdot t))] \\
\text { if } e \in E \text { and } 1 / 2 \leqq \alpha(e) \cdot t \leqq 1 \\
\text { if } e \in E^{\prime},
\end{array}\right.
$$




$$
\bar{K}(e, t)=\left\{\begin{array}{c}
\nu[D(D(e, 2 \alpha(e) \cdot t), 2 \alpha(D(e, 2 \alpha(e) \cdot t)) \cdot t)] \text { if } \\
e \in E, 0 \leqq \alpha(e) \cdot t \leqq 1 / 2 \text { and } \\
0 \leqq \alpha(D(e, 2 \alpha(e) \cdot t)) \cdot t \leqq 1 / 2 \\
\nu[G(D(D(e, 2 \alpha(e) \cdot t), 1), 2-2 \alpha(D(e, 2 \alpha(e) \cdot t)) \cdot t)] \text { if } \\
e \in E, 0 \leqq \alpha(e) \cdot t \leqq 1 / 2 \text { and } \\
1 / 2 \leqq \alpha(D(e, 2 \alpha(e) \cdot t)) \cdot t \leqq 1 \\
\nu[G(D(e, 1), \beta(e, t))] \text { if } e \in E \text { and } 1 / 2 \leqq \alpha(e) \cdot t \leqq 1 \\
\nu(e) \text { if } e \in E^{\prime} .
\end{array}\right.
$$

$\beta(e, t)=\min \left\{4 \alpha(e) \cdot t-2 \alpha(e) \cdot t^{2}-t, 1\right\}$ in the preceding definition of $\bar{K}$.

\section{Applications.}

Given $\xi=(E, p, B), \eta=\left(E^{\prime}, p^{\prime}, B\right)$ and $f: \xi \rightarrow \eta$ we will denote by $\mu_{f}=\left(M_{f}, q, B\right)$ the space over $B$ with total space the mapping cylinder of $f$, and $q$ the map induced by $p^{\prime}$ and $\bar{p}: E \times I \rightarrow B$ where $\bar{p}(e, t)=p(e)$. The following theorem is an immediate corollary to Theorem (2.5).

THEOREM (3.1). Let $B$ be a paracompact, locally compact space with the property that $(B \times B, \Delta B)$ is a closed cofibration. Then if $\xi$ and $\eta$ are fibrations over $B$, and if $f: \xi \rightarrow \eta$ is a fiber preserving $\operatorname{map}, \mu_{f}$ is a fibration.

The notion of strong fiber homotopy equivalence (due to P. Tulley) is studied and used extensively by P. Tulley in [9] and S. Langston in [6].

Definition (3.2). Two fibrations $\xi=(E, p, B)$, and $\eta=\left(E^{\prime}, p^{\prime}, B\right)$ have the same strong fiber homotopy type denoted $\xi \sim_{s} \eta$ if there is a fibration $\Gamma=\left(E^{*}, p^{*}, B \times I\right)$ with $\Gamma_{B \times\{0\}}=\xi$ and $\Gamma_{B \times\{1\}}=\eta$.

In [6] Langston proved that fiber homotopy equivalence is the same as strong fiber homotopy equivalence when $E$ and $E^{\prime}$ are separable metric ANR's. In [9] Tulley proved that if $f: \xi \rightarrow \eta$ is a fiber homotopy equivalence of fibrations, and if $\mu_{f}$ is a fibration then $\xi \sim_{s} \eta$. The result in [9] however, assumed $E$ was compact to show $\mu_{f}$ is a fibration. By Theorem (3.1) and Theorem 11 in [9] we then have the following result.

THEOREM (3.3). If $B$ is paracompact, locally compact and $(B \times B, \Delta B)$ a closed cofibration, then for any two fibrations $\xi$ and $\eta, \xi \sim \eta$ if and only if $\xi \sim s \eta$. 
In [6] Langston used the relationship between strong fiber homotopy equivalence and fiber homotopy equivalence to consider the problem of extending a fibration.

Definition (3.4). If $A \subset B$ and $\xi=(E, p, A)$ is a fibration, then a fibration $\eta$ is an extension of $\xi$ if $\eta_{A}=\xi$.

Langston in [6] proved that if $(B, A)$ is a finite $C W$ pair, and if two fibrations $\xi$ and $\xi^{\prime}$ over $A$ with ANR total spaces are fiber homotopy equivalent, then $\xi$ can be extended to $B$ if and only if $\xi^{\prime}$ extends to $B$. The next theorem will generalize this result. We prove a lemma first to give a better statement of the theorem.

Lemma (3.5). If $(X, A)$ and $(X \times X, \Delta X)$ are closed cofibrations, $A$ metric, then $(A \times A, \Delta A)$ is a closed cofibration.

Proof. Let $D: X \times I \rightarrow X, \phi: X \rightarrow I$ be associated with the pair $(X, A)$ and $\bar{D}: X \times X \times I \rightarrow X \times X, \bar{\phi}: X \times X \rightarrow I$ with the pair $(X \times X, \Delta X)$ as in Lemma (2.1). Let $V=\phi^{-1}(0,1] \subset X$ and $r: V \rightarrow A$ be the retraction $r(x)=D(x, 1)$. Let $U=\bar{\phi}^{-1}(0,1) \subset X \times X$, and define $\sigma: U \rightarrow X^{I}$ as in Lemma (2.3) using $\bar{D}$ and $\bar{\phi}$.

Let $W$ be a relatively open set in $A \times A$ with the property that $\sigma^{-1}(V) \cap A \times A \supset \bar{W} \supset W \supset \Delta A . \quad(\bar{W}=$ closure of $W$.$) We then have$ $\sigma(x, y)(t) \in V$ for all $(x, y) \in \bar{W}$. Choose $\alpha: A \times A \rightarrow I$ such that $\alpha^{-1}(1)=$ $\triangle A$ and $\alpha(A \times A-W)=0$. We define $\hat{D}: A \times A \times I \rightarrow A \times A$ and $\hat{\phi}: A \times A \rightarrow I$ as follows:

$$
\begin{gathered}
\hat{D}(x, y, t)=\left\{\begin{array}{l}
(x, y) \quad \text { if }(x, y) \in A \times A-W \\
(r \sigma(x, y)(\alpha(x, y) \cdot t), r \sigma(x, y)(1-\alpha(x, y) \cdot t)) \\
\text { if } 0 \leqq \alpha(x, y) \leqq 1 / 2 \text { and } \quad(x, y) \in \bar{W} \\
(r \sigma(x, y)(1 / 2 \cdot t), r \sigma(x, y)(1-1 / 2 \cdot t))
\end{array}\right. \\
\hat{\phi}(x, y)= \begin{cases}0 & \text { if } 1 / 2 \leqq \alpha(x, y) \leqq 1 \text { and } \quad(x, y) \in \bar{W}, \\
2 \alpha(x, y)-1 & \text { if } 1 / 2 \leqq \alpha(x, y) \leqq 1 .\end{cases}
\end{gathered}
$$

Using $\hat{D}$ and $\hat{\phi}$, Lemma (2.1) shows that $(A \times A, \Delta A)$ is a closed cofibration.

Theorem (3.6). Let $B$ be a metric space such that $(B \times B, \Delta B)$ is a cofibration, and $A$ a locally compact subset of $B$ with $(B, A) a$ cofibration. Then if $\xi$ and $\eta$ are fiber homotopy equivalent fibrations over $A, \xi$ can be extended to $B$ if and only if $\eta$ extends to $B$.

Proof. $\xi=(E, p, A)$ and $\eta=\left(E^{\prime}, p^{\prime}, A\right)$ are strongly fiber homotopy equivalent by Theorem (3.3), therefore there is a fibration $\Gamma=$ 
$\left(E^{*}, p^{*}, A \times I\right)$ with $\Gamma_{A \times\{0\}}=\xi$ and $\Gamma_{A \times\{1\}}=\eta$. If $\xi$ has an extension $\bar{\xi}=(\bar{E}, \bar{p}, B)$, consider the space over $B \times\{0\} \cup A \times I$

$$
\bar{\xi} \cup \Gamma=\left(\bar{E} \cup E^{*}, \bar{p} \cup p^{*}, B \times\{0\} \cup A \times I\right) .
$$

Since $(A \times I, A \times\{0\})$ and $(B \times\{0\}, A \times\{0\})$ are closed cofibrations, Theorem (4.2) of [1] applies, and $\bar{\xi} \cup \Gamma$ is a fibration. Let $r: B \times I \rightarrow B \times\{0\} \cup A \times I$ be a retraction. Then $r^{*}(\bar{\xi} \cup \Gamma)$, the pullback of $\xi \cup \Gamma$ by $r$, is a fibration, and the desired extension of $\eta$ is given by $r^{*}(\bar{\xi} \cup \Gamma)_{B \times\{1}$.

REMARK. Note that the extension of $\eta$ given by this theorem is fiber homotopy equivalent to the extension of $\xi$ that we started with.

We now apply Theorem (3.6) and Theorem (4.2) of [1] to prove a stronger form of Dold's pasting lemma. (See $\S 6.4$, of [2].)

TheOREm (3.7). Let $X$ be a metric space of the form $X=X_{1} \cup X_{2}$ with $X_{1} \cap X_{2}$ locally compact, and $\left(X_{i}, X_{1} \cap X_{2}\right)$ and $\left(X_{i} \times X_{i}, \Delta X_{i}\right)$ cofibrations $i=1,2$. Then if $\xi^{1}=\left(E_{1}, p_{1}, X_{1}\right)$ and $\xi^{2}=\left(E_{2}, p_{2}, X_{2}\right)$ are fibrations such that $\xi_{X_{1} \cap X_{2}}^{1} \sim \xi_{X_{1} \cap X_{2}}^{2}$, there is a fibration $\eta=\left(E, p, X_{1} \cup X_{2}\right)$ with $\eta_{x_{1}}=\xi^{1}$ and $\eta_{x_{2}} \sim \xi^{2}$.

Proof. $\xi_{X_{1} \cap X_{2}}^{1} \sim \xi_{X_{1} \cap x_{2}}^{2}$ and $\xi_{X_{1} \cap x_{2}}^{2}$ extends to $\xi^{2}$ over $X_{2}$, therefore by Theorem (3.6), there is an extension $\xi=\left(\bar{E}, \bar{p}, X_{2}\right)$ of $\xi_{X_{1} \cap X_{2}}^{1}$ to $X_{2}$ which is fiber homotopy equivalent to $\xi^{2}$. We now let

$$
\eta=\left(\bar{E} \cup \bar{E}_{1}, \bar{p} \cup p_{1}, X_{1} \cup X_{2}\right) \text {. }
$$

$\eta$ is fibration by Theorem (4.2) of [1] and $\eta_{X_{1}}=\xi^{1}, \eta_{x_{2}} \sim \xi^{2}$ by construction.

As a final application of Theorem (2.5) we consider the problem of attaching a disk bundle to a fibration by a fiber preserving map on the associated sphere boundle. Ibisch in [5] studied this problem for weak fibrations i.e. triples with the weak covering homotopy property. If $\xi$ is a disk bundle over $B, B$ as in Theorem (2.5), it is clear that at least locally, the total space of the associated sphere bundle $\xi_{0}$ is a closed cofibration in the total space of $\xi$. Therefore given a fibration $\eta$ over $B$ and a fiber preserving map $f: \xi_{0} \rightarrow \eta$, we can apply Theorem (2.5) locally to get $\xi \cup_{f} \eta$ is a fibration.

REMARK. Using Theorem 2 of [3] one can show in fact that the inclusion of the total space of $\xi_{0}$ in the total space of $\xi$ is a closed cofibration.

By a different method we can eliminate the condition $(B \times B, \Delta B)$ is a closed cofibration. Let 


$$
\xi=\left(E_{1} \cup E_{2}, p, B\right), \xi^{1}=\left(E_{1}, p_{1}, B\right), \xi^{2}=\left(E_{2}, p_{2}, B\right)
$$

and

$$
\xi^{0}=\left(E_{1} \cap E_{2}, p_{0}, B\right)=\left(E_{0}, p_{0}, B\right)
$$

with $p_{i}=p \mid E_{i}$.

Lemma (3.8). If $\left(E_{i}, E_{0}\right) i=1,2$ are closed cofibrations and $\xi^{0}, \xi^{1}, \xi^{2}$ are fibrations, then $\xi$ is a fibration.

Proof. Choose a lifting function $\lambda_{0}$ for $\xi^{0}$. By Lemma (2.4) we can find lifting functions $\lambda_{i}: \Omega_{p_{i}} \rightarrow E_{i}^{I} i=1$, 2, for $\xi^{2}$ whose restrictions to $\Omega_{p_{0}}$ are $\lambda_{0}$. A lifting function for $\xi$ is then defined by

$$
\lambda(e, \omega)=\left\{\begin{array}{lll}
\lambda_{1}(e, \omega) & \text { if } & (e, \omega) \in \Omega_{p_{1}} \\
\lambda_{2}(e, \omega) & \text { if } \quad(e, \omega) \in \Omega_{p_{2}}
\end{array}\right.
$$

Now let $\xi=(E, p, B)$ be a disk bundle with $\dot{\xi}=(\dot{E}, \dot{p}, B)$ the boundary sphere bundle, and $\eta=\left(E^{\prime}, p^{\prime}, B\right)$ a fibration.

THEOREM (3.9). Let $B$ be a paracompact, locally compact space and $f: \dot{\xi} \rightarrow \eta$. Then $\xi \cup_{f} \eta$ is a fibration.

Proof. Consider the mapping cylinder $\mu_{f}=\left(M_{f}, \bar{q}, B\right)$. Given $b_{0} \in B$, we can choose a neighborhood $W$ of $b_{0}$ with compact closure, so that $\dot{\xi}$ is trivial over $\bar{W} . E_{\bar{W}}$ is then compact, therefore we can apply. Theorem (8) in [9] to prove $\mu_{f}$ is a fibration over $\bar{W} . \mu_{f}, \xi$, and $\xi$ is thus a fibration by the Uniformization Theorem. Letting $\|\cdot\|$ denote the norm induced by a Riemannian metric on $\xi$, we define $E_{i} \subset E \cup_{f} E^{\prime}$ as follows:

$$
\begin{aligned}
& E_{1}=\{e \in E \mid\|e\| \geqq 1 / 2\} \cup_{f} E^{\prime} \\
& E_{2}=\{e \in E \mid\|e\| \leqq 1 / 2\} .
\end{aligned}
$$

Then $\left(E_{1}, q \mid E_{1}, B\right),\left(E_{2}, q \mid E_{2}, B\right)$ and $\left(E_{1} \cap E_{2}, q \mid E_{1} \cap E_{2}, B\right)$ are fiberwise homeomorphic to $\mu_{f}, \xi$, and $\dot{\xi}$ respectively and are thus fibrations. $\left(E_{i}, E_{1} \cap E_{2}\right)$ is a closed cofibration $i=1,2$. Therefore Lemma (3.8) applies, and $\xi \cup_{f} \eta$ is a fibration.

\section{REFERENCES}

1. J. Arnold, Local to global theorems in the theory of Hurewicz fibrations, Trans. Amer. Math. Soc., 164 (1972), 179-188.

2. A. Dold, Halbexacte Homotopie Funktoren, Lecture Notes in Mathematics 12, SpringerVerlag 1966.

3. - Die Homotopieerweiterungseigenschaft ist eine lokale Eigenschaft, Inventiones Math., 6 (1968), 185-189. 
4. W. Hurewicz, On the concept of fibre space, Proc. Nat. Acad. Sci. U.S.A., 41 (1955), 956-961.

5. H. D. Ibisch, Anheften von Kugelbündeln an Faserraüme, Math. Zeitschr., 91 (1966), 294-299.

6. S. L. Langston, Replacement and extension theorems in the theory of Hurewicz fiber spaces, Ph. D. Thesis, Univ. of Wisconsin (1968).

7. E. Spanier, Algebraic Topology, McGraw Hill (1966).

8. A Str $\phi \mathrm{m}$, Note on cofibrations II, Math. Scand., 22 (1968), 130-142.

9. P. Tulley, On strong homotopy equivalence and extensions for Hurewicz fibrations, Duke Math. J., 36 (1969), 609-619.

10. J.H.C. Whitehead, Note on a theorem due to Borsuk, Bull. Amer. Math. Soc., 54 (1948), 1125-1132.

11. G. S. Young, $A$ condition for the absolute homotopy extension property, Amer. Math. Monthly, 71 (1964), 896-897.

Received March, 27, 1972 and in revised form January 3, 1973.

UNIVERSITY OF WISCONSIN-MILWAUKEE 



\section{PACIFIC JOURNAL OF MATHEMATICS}

\section{EDITORS}

D. Gilbarg and J. Milgram

Stanford University

Stanford, California 94305

\author{
R. A. Beaumont \\ University of Washington \\ Seattle, Washington 98105
}

J. DuGUNDJI

Department of Mathematics

University of Southern California

Los Angeles, California 90007

Richard ARENS

University of California

Los Angeles, California 90024

\section{ASSOCIATE EDITORS}
E. F. BECKENBACH
B. H. NeumanN
F. WOLF
K. YosHIDA

\section{SUPPORTING INSTITUTIONS}

UNIVERSITY OF BRITISH COLUMBIA

CALIFORNIA INSTITUTE OF TECHNOLOGY

UNIVERSITY OF CALIFORNIA

MONTANA STATE UNIVERSITY

UNIVERSITY OF NEVADA

NEW MEXICO STATE UNIVERSITY

OREGON STATE UNIVERSITY

UNIVERSITY OF OREGON

OSAKA UNIVERSITY

\author{
UNIVERSITY OF SOUTHERN CALIFORNIA \\ STANFORD UNIVERSITY \\ UNIVERSITY OF TOKYO \\ UNIVERSITY OF UTAH \\ WASHINGTON STATE UNIVERSITY \\ UNIVERSITY OF WASHINGTON
* * * *
AMERICAN MATHEMATICAL SOCIETY \\ NAVAL WEAPONS CENTER
}

The Supporting Institutions listed above contribute to the cost of publication of this Journal, but they are not owners or publishers and have no responsibility for its content or policies.

Mathematical papers intended for publication in the Pacific Journal of Mathematics should be in typed form or offset-reproduced, (not dittoed), double spaced with large margins. Underline Greek letters in red, German in green, and script in blue. The first paragraph or two must be capable of being used separately as a synopsis of the entire paper. The editorial "we" must not be used in the synopsis, and items of the bibliography should not be cited there unless absolutely necessary, in which case they must be identified by author and Journal, rather than by item number. Manuscripts, in duplicate if possible, may be sent to any one of the four editors. Please classify according to the scheme of Math. Rev. Index to Vol. 39. All other communications to the editors should be addressed to the managing editor, Richard Arens, University of California, Los Angeles, California, 90024.

50 reprints are provided free for each article; additional copies may be obtained at cost in multiples of 50 .

The Pacific Journal of Mathematics is issued monthly as of January 1966. Regular subscription rate: $\$ 48.00$ a year (6 Vols., 12 issues). Special rate: $\$ 24.00$ a year to individual members of supporting institutions.

Subscriptions, orders for back numbers, and changes of address should be sent to Pacific Journal of Mathematics, 103 Highland Boulevard, Berkeley, California, 94708.

PUBLISHED BY PACIFIC JOURNAL OF MATHEMATICS, A NON-PROFIT CORPORATION

Printed at Kokusai Bunken Insatsusha (International Academic Printing Co., Ltd.), 270, 3-chome Totsuka-cho, Shinjuku-ku, Tokyo 160, Japan. 


\section{Pacific Journal of Mathematics}

\section{Vol. 46, No. 2 December, 1973}

Christopher Allday, Rational Whitehead products and a spectral sequence of

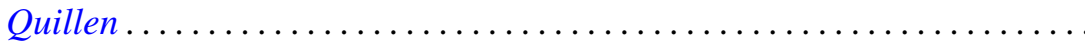

James Edward Arnold, Jr., Attaching Hurewicz fibrations with fiber

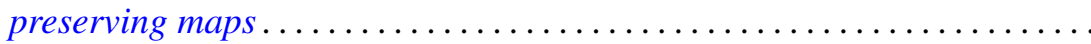

Catherine Bandle and Moshe Marcus, Radial averaging transformations with various metrics.................................

David Wilmot Barnette, A proof of the lower bound conjecture for convex

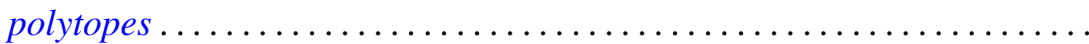

Louis Harvey Blake, Simple extensions of measures and the preservation of

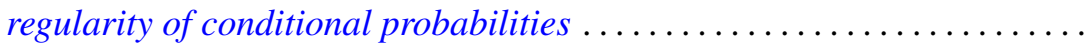

James W. Cannon, New proofs of Bing's approximation theorems for

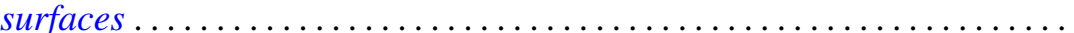

C. D. Feustel and Robert John Gregorac, On realizing HNN groups in

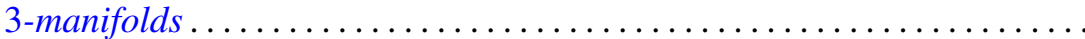

Theodore William Gamelin, Iversen's theorem and fiber algebras . . . . . . . . 389

Daniel H. Gottlieb, The total space of universal fibrations . . . . . . . . . . . .

Yoshimitsu Hasegawa, Integrability theorems for power series expansions of

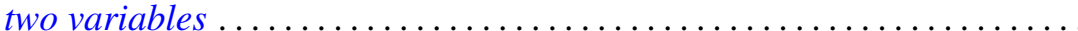

Dean Robert Hickerson, Length of period simple continued fraction expansion of $\sqrt{ } d$

Herbert Meyer Kamowitz, The spectra of endomorphisms of the disc algebra.

Dong S. Kim, Boundedly holomorphic convex domains

Daniel Ralph Lewis, Integral operators on $\mathscr{L}_{p}$-spaces ...

John Eldon Mack, Fields of topological spaces . . . . . . . . .

V. B. Moscatelli, On a problem of completion in bornology

Ellen Elizabeth Reed, Proximity convergence structures. .

Ronald C. Rosier, Dual spaces of certain vector sequence spaces .

Robert A. Rubin, Absolutely torsion-free rings

Leo Sario and Cecilia Wang, Radial quasiharmonic functions . .

James Henry Schmerl, Peano models with many generic classes .

H. J. Schmidt, The $\mathscr{F}$-depth of an $\mathscr{F}$-projector ............

Edward Silverman, Strong quasi-convexity. . . . . . . . . . . . . . . . . 549

Barry Simon, Uniform crossnorms ....................... 555

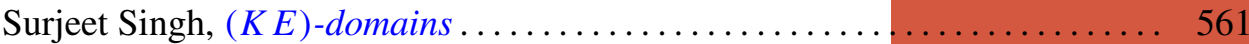

Ted Joe Suffridge, Starlike and convex maps in Banach spaces . . . . . . . . 575

Milton Don Ulmer, $C$-embedded $\Sigma$-spaces . . . . . . . . . . . . . . . . 591

Wolmer Vasconcelos, Conductor, projectivity and injectivity . . . . . . . . . 603 